\title{
EL PAPEL DE LA INTERCULTURALIDAD EN LA ENSEÑANZA DE LAS CIENCIAS NATURALES: LA ZOOTERAPIA COMO ESTUDIO DE CASO
}

\section{THE ROLE OF INTERCULTURALITY IN THE TEACHING OF NATURAL SCIENCES: ZOOTHERAPY AS A CASE STUDY}

\author{
Mauricio VARGAS-CLAVIJO ${ }^{1 *}$; Alondra FLORES-SILVA²; Geilsa BAPTISTA ${ }^{3}$
}

${ }^{1}$ Estudiante del Doctorado Interinstitucional en Educación y Pedagogía, Universidad del Valle, Colombia, *antroelitrus@ gmail.com; ${ }^{2}$ Estudiante del Doctorado Ciencias en Biosistemática, Ecología y Manejo de Recursos Naturales y Agrícolas (BEMARENA), Universidad de Guadalajara, México, alondra.floresilva@gmail.com; ${ }^{3}$ Departamento de Educação, Universidade Estadual de Feira de Santana, Brasil, geilsa@uefs.br

\section{RESUMEN}

Submitted: 22/07/2020; Accepted: 24/09/2020

Las prácticas zooterapéuticas son parte importante del cuidado de la salud en muchas zonas rurales y urbanas de Colombia, sin embargo, estos saberes y prácticas son difusamente contemplados dentro de las competencias y aprendizajes asociados al ambiente por parte del sistema educativo nacional. El objetivo de esta investigación fue documentar los conocimientos zooterapéuticos de los estudiantes y sus familias de dos escuelas urbanas de El Yopal, Colombia para poder generar una discusión dirigida a la pertinencia de una enseñanza intercultural para la enseñanza de ciencias. Encontramos que tanto los estudiantes como sus familias emplean 32 etnoespecies para tratar 43 enfermedades, lo que se traduce en la importancia de reconocer la posibilidad de llevar a cabo un diálogo intercultural dentro del salón de clases donde se parta del respeto a las otras formas de conocimiento diferentes al científico, donde el salón de clases constituya un espacio de intercambio de ideas cuyos conocimientos tradicionales puedan ser compartidos y no anulados o excluidos. La complementariedad de conocimientos tradicionales y científicos puede ayudar a los estudiantes a ampliar su universo de conocimientos y valorar los diferentes modos de solucionar padecimientos de salud con la posibilidad de aplicarlos en su contexto sociocultural.

Palabras clave: Conocimiento zooterapéutico de los estudiantes, diálogo intercultural, enseñanza de las ciencias.

\begin{abstract}
Zootherapeutic practice are an important part of health care in many rural and urban areas of Colombia, nevertheless, these knowledges and practices are difussely contemplated within the competencies and learning associated with environment by the national educational system. The objective of this research was to document the zootherapeutic knowledge of the students and their families from two urban schools in El Yopal, Colombia in order to generate a discussion aimed at the relevance of intercultural teaching for science education. We record that both students and their families employ 32 ethnospecies to treat 43 diseases, which translates into the importance to recognize the possibility of carry out an intercultural dialogue in the classroom where you start from respect for other forms of knowledge in addition to the scientist, where the classroom establish the space for the exchange of ideas whose traditional knowledge can be shared and not invalidate or excluded. The complementary of traditional and school scientific knowledge can help students to expand their universe of knowledge and asses the different ways of solving health conditions with the possibility of applying them in their sociocultural context.
\end{abstract}

Keywords: Zootherapeutic knowledge of students, intercultural dialogue, science teaching.

\section{INTRODUCCIÓN}

Teniendo en cuenta la alta riqueza étnica y cultural de los países latinoamericanos y caribeños, los currículos en ciencias deben transformarse epistémica y metodológicamente con el objetivo de convertir a la ciencia en un sistema de conocimiento al alcance del pueblo y no en un paradigma al que sólo pueden acceder selectos grupos académicos y sociales. Los sistemas de conocimiento local, ancestral, tradicional e indígena deben tomarse en cuenta no sólo en el discurso político educativo de estos países, sino también en los documentos que orientan el diseño curricular a diferentes escalas, con el ánimo de 
demostrarse que en realidad se está en esa búsqueda de una verdadera educación inclusiva social y culturalmente (BALL y BOWE 1992; CANEN, 2000; SILVA, 2003).

En el caso particular del sistema educativo colombiano, en los referentes nacionales de calidad educativa las áreas de Ciencias Sociales y Ciencias Naturales en los niveles de preescolar, básica y media, se contemplan difusamente competencias y aprendizajes relacionados con saberes y prácticas ancestrales y tradicionales asociadas al ambiente, o por lo menos, esto es lo que permite dar a entender cuando se leen los Estándares Básicos de Competencias ${ }^{1}$ (EBC) en Ciencias Naturales y Ciencias Sociales (MINISTERIO DE EDUCACIÓN NACIONAL, 2004), los Derechos Básicos de Aprendizaje ${ }^{2}$ (DBA) y las Matrices de Referencia ${ }^{3}$ (MR) de Ciencias Naturales (MINISTERIO DE EDUCACIÓN NACIONAL, 2016a; 2016b) y los Derechos Básicos de Aprendizaje en Ciencias Sociales (MINISTERIO DE EDUCACIÓN NACIONAL, 2016c).

En los EBC de Ciencias Naturales en el conjunto de grados octavo y noveno, en el manejo de conocimientos asociados a la historia y las culturas, los estudiantes deben alcanzar el estándar: "Reconocer los aportes artísticos y científicos de los grupos étnicos antiguos y actuales en la construcción de la identidad colombiana". En los DBA de Ciencias Naturales, sólo en grado undécimo, una de las "evidencias de aprendizaje" del quinto DBA: "Identifica las implicaciones que tiene para Colombia, en los ámbitos social, ambiental y cultural el hecho de ser "un país megadiverso". En las MR de Ciencias Naturales, en el entorno "Ciencia, Tecnología y Sociedad" de noveno grado, a través de un "aprendizaje" se busca "Comprender que existen diversos recursos y analizar su impacto sobre el entorno cuando son explotados, así como las posibilidades de desarrollo para las comunidades".

Por su parte, en los DBA de Ciencias Sociales, es posible que sea más fácil identificar expresiones de la cultura local, sin embargo, este enfoque cultural se desarrolla desde una perspectiva más amplia y no sólo desde lo ambiental. Por citar algunos ejemplos, en grado primero una de las "evidencias de aprendizaje" del quinto DBA que trata de la identidad individual, busca que el estudiante: reconozca “...su individualidad y su pertenencia a los diferentes grupos sociales"; a través de "... las costumbres y tradiciones culturales de su comunidad mediante los relatos de los abuelos y personas mayores del barrio, vereda o lugar donde vive". En grado tercero, todas las "evidencias de aprendizaje" señaladas en el sexto DBA, favorecen que el estudiante analice "...las contribuciones de los grupos humanos que habitan en su departamento, municipio o lugar donde vive, a partir de sus características culturales: lengua, organización social, tipo de vivienda, cosmovisión y uso del suelo". Algo similar sucede con el cuarto DBA del cuarto grado de primaria, dado que permite que el estudiante "Analice las características de las culturas ancestrales que, a la llegada de los españoles, habitaban el territorio nacional".

Si bien esta situación debería revisarse porque evidencia la inexistencia de una política nacional sobre enseñanza de las ciencias con enfoque inclusivo, diferencial e intercultural, vale la pena reconocer que el Ministerio de Educación Nacional, otras entidades del Estado y las mismas comunidades, han venido impulsado iniciativas en materia de política pública para la atención de la diversidad poblacional con programas bajo esquemas de inclusión social, étnica y cultural. Un ejemplo de este salto político tiene que ver con la perspectiva diferencial que se viene implementando en los Proyectos Educativos de cada centro educativo del país (MINISTERIO DE EDUCACIÓN NACIONAL, 2010; CONTCEPI, 2012; MINISTERIO DEL INTERIOR, 2014). Lo que se persigue, es que las comunidades no continúen desarrollando proyectos educativos que no les son propios bajo una estructura Estado-centrista, sino proyectos educativos que realmente estén fundamentados en sus planes de vida individual y comunitario.

Las propuestas pasan de ser simplemente ideas educativas innovadoras culturalmente a convertirse en el resultado de un proceso constructivo colectivo endógeno, posibilitando mayor visibilidad y empoderamiento de las comunidades locales en sus políticas y formas de direccionar la educación. En varias regiones del país ya se han comenzado a implementar Proyectos de Educación Propia, Educación Indígena, Educativos Comunitarios, Educativos Campesinos, y más recientemente, Educativos

\footnotetext{
1 Son criterios claros y públicos que permiten identificar lo que deben aprender los estudiantes a lo largo de su vida escolar en básica y media, y en los que se establecen puntos de referencia de lo que están en capacidad de saber y saber hacer, en cada una de las áreas y niveles (Ministerio de Educación Nacional, 2004).

2 Son aprendizajes estructurantes para un grado y un área particular, entendiéndose como aprendizaje la agrupación de conocimientos, habilidades y actitudes que se desarrollan en un contexto cultural e histórico de la persona. Son estructurantes porque expresan las unidades básicas y fundamentales sobre las cuales se puede construir el sujeto (Ministerio de Educación Nacional, 2016c).

${ }^{3}$ La Matriz de Referencia (MR) es una tabla basada en los Estándares Básicos de Competencias (EBC), organizada en dos entradas (x,y) y en la que se establece la relación entre competencias (uso de conceptos, explicación de fenómenos e indagación) y componentes (entornos vivo, físico, químico, y ciencia, tecnología y sociedad). Ha servido como herramienta curricular para relacionar los aprendizajes que evalúa el ICFES en cada competencia, relacionándolo con las evidencias de lo que debería conseguir un estudiante al finalizar cada grupo de grados (Ministerio de Educación Nacional, 2016b).
} 
Interculturales. Con este gran paso no sólo se ha venido consiguiendo que el gobierno y la sociedad mayoritaria reconozcan y valoren los derechos de los pueblos culturalmente diferenciados, sino que también se generen avances para el establecimiento de un sistema de educación propio o intercultural el cual ha sido amparado en el Plan Sectorial de Educación del Plan Nacional de Desarrollo 2010-2014 (MISNITERIO DE EDUCACIÓN NACIONAL, 2010; CONTCEPI, 2012).

Aun cuando todavía falta camino por recorrer, la experiencia pedagógica intercultural en la enseñanza de las ciencias en instituciones educativas urbanas y rurales, sigue evidenciándose tímidamente. Los enfoques principalmente siguen dos trayectorias: la primera, en la que los saberes tradicionales asociados al ambiente son incorporados a ciertos temas de áreas obligatorias como las ciencias naturales y sociales; y la segunda, donde se investiga sobre estos conocimientos través de "proyectos pedagógicos transversales" o "proyectos de aula". Esta tendencia curricular en la enseñanza de las ciencias con enfoque cultural, también fue evidenciada por Verde et al. (2009) en instituciones educativas españolas frente a la enseñanza de la etnobotánica en la escuela básica.

Docentes y estudiantes trabajan en sus clases temas como el empleo tradicional y popular de plantas medicinales y aromáticas, recuperación de semillas nativas, formas de producción agropecuaria tradicional, elaboración de platos culinarios tradicionales, entre otras temáticas. Por citar algunos casos, estudiantes, docente y familiares del Centro Educativo Rural Llano Grande, sede Laguna en Chítaga, Norte de Santander, cultivaron plantas medicinales y aromáticas para uso de la misma comunidad (MORENO, 2012). Alumnos del Centro Educativo Rural El Alisal en Guacamayas, Boyacá, como parte del proyecto comunitario "Etnobotánica de plantas medicinales, aromáticas y alimenticias de Guacamayas, Boyacá, Colombia" ayudaron a construir un herbario y cultivaron especies de plantas medicinales en su institución (BELTRÁN-CUARTAS et al., 2010).

Partiendo de este panorama, y en la búsqueda de incorporar la enseñanza de las ciencias con un enfoque epistémico local e inclusivo social y culturalmente en el sistema educativo, se presentan los resultados de un estudio exploratorio con estudiantes de dos escuelas públicas urbanas de Yopal, Casanare, Colombia, en relación con el empleo de animales como medicina (zooterapia). Se realizó un análisis y discusión acerca de la necesidad de dar un cambio epistemológico y didáctico a la enseñanza de las ciencias a partir de un abordaje intercultural, además de colocar en evidencia la necesidad de acercar más a las familias y comunidad a los centros educativos en los procesos de enseñanza de las ciencias, y la de renovar los currículos en ciencias con el propósito de enriquecer el diálogo intercultural en las aulas de clase.

\section{METODOLOGÍA}

\section{Área de estudio y aspectos socioculturales}

El estudio se desarrolló con la participación de niños y adolescentes de todos los géneros, entre 8 y 14 años de dos instituciones educativas públicas urbanas: "El Paraíso" y "Carlos Lleras" del municipio El Yopal, capital del departamento de Casanare, Colombia. El Yopal, o "Yopal" como usualmente se le conoce, se encuentra ubicado sobre la margen sur del río Cravo Sur. Ocupa una extensión de $2.271 \mathrm{~km}^{2}$ y una altitud máxima de 350 m.s.n.m. Al Norte limita con Labranzagrande y Paya, municipios del departamento de Boyacá; al Oriente, con los municipios de Nunchía y San Luís de Palenque; al Sur con Maní y Orocué; y al Occidente, con Aguazul y Pajarito, Boyacá. Dista de 387 km. de Bogotá, Distrito Capital de la República de Colombia por la vía que conduce hacia el oriente del país. La temperatura promedio oscila entre $26^{\circ}$ y $28^{\circ}$ centígrados y una precipitación media anual aproximada de $2.500 \mathrm{~mm}$. El clima es característico de una zona húmeda-tropical (VALLEJO, 2011).

Se caracteriza por ser un municipio relativamente nuevo en su formación administrativa y política, y por su acelerado crecimiento económico y poblacional debido al importante desarrollo de la industria petrolera en la región. Se ha constituido como la segunda ciudad más importante de la Orinoquía colombiana después de Villavicencio, Meta. Administrativamente, pasa de ser un caserío adscrito al municipio de Marroquín en 1931, a reconocerse como Inspección de Policía en 1934, corregimiento en 1936 y, por último, como municipio en 1942, erigiéndose como capital en el año de 1973 (ROJAS et al., 1997). Su economía se basa principalmente en la explotación petrolera, comercio, administración pública y poco a poco la diversificación de la industria agrícola siendo la ganadería vacuna e industria arrocera, los principales renglones económicos.

Según Dureau y Goueset (2001), Casanare se caracterizaba por ser una región de sabanas poco pobladas que durante mucho tiempo constituyeron las áreas de colonización agraria, donde la ganadería 
extensiva y los cultivos regados eran las principales fuentes económicas. Hacia 1985 era poco urbanizado e industrializado y el sector agrícola representaba dos terceras partes del PIB departamental.

El estudio se desarrolló en dos Escuelas Públicas de Yopal durante el año 2011, las cuales fueron parte de la implementación del modelo educativo flexible Círculos de Aprendizaje, donde el autor principal de la investigación se desempeñaba como coordinador del Programa. El conocimiento general de la población escolar, similitud en características sociodemográficas de ambas escuelas y la relación cercana con los estudiantes y directivos del colegio, motivaron que estas dos instituciones fuera elegidas como objetivo de investigación. Los estudiantes de ambos centros educativos se caracterizan por provenir de familias de escasos recursos económicos, bajos niveles de escolaridad y alta inestabilidad laboral. Las madres de los estudiantes por lo general se ocupan como "amas de casa" mientras que los padres se dedican al trabajo informal u oficios varios. Muchos son obreros de construcción, empleados de talleres mecánicos, electricistas, auxiliares, vigilantes o contratistas ocasionales de compañías, la gobernación o la alcaldía municipal (INSTITUCIÓN EDUCATIVA EL PARAÍSO, 2010). También están dedicados a laborar en empresas unifamiliares como modistería, salas de belleza, tiendas barriales, ornamentación y carpintería (INSTITUCIÓN EDUCATIVA CARLOS LLERAS RESTREPO, 2011). El 42\% de los estudiantes son originarios de Yopal u otro municipio de Casanare mientras que el 58\% restante son de familias colonizadoras provenientes de los departamentos de Meta, Arauca, Boyacá, Santander, Cundinamarca o de otras regiones del país.

\section{Procedimientos metodológicos}

Para llevar a cabo la investigación se colocaron en conocimiento de los directivos docentes y docentes del área de Ciencias Naturales y Educación Ambiental de ambas instituciones educativas los objetivos y alcances del estudio. Igualmente, se contó con la autorización de los padres de familia de los estudiantes quienes aceptaron que se llevara a cabo con fines investigativos - educativos. La autorización de los padres se diligenció previamente en un formulario entregado a los estudiantes quienes lo llevaban a sus hogares para ser leído y firmado.

Una vez obtenidos los consentimientos correspondientes de parte de sus acudientes y los docentes y directivos docentes, se suministraron 173 cuestionarios a igual número de estudiantes, dejándose una semana para responderlo. Con la ayuda de sus familiares, respondieron las siguientes preguntas: a) ¿emplean animales como medicina?; b) ¿qué parte(s) son utilizada(s)?; c) ¿y para tratar qué padecimiento o enfermedad?; d) ¿cuál es el modo de empleo?; e) ¿alguna vez ha empleado este animal o parte del animal como medicamento?

El desarrollo del cuestionario se argumentó como una tarea complementaria al desarrollo del plan de estudios en el área de Ciencias Naturales y Educación Ambiental, materia de Biología. Al no estar directamente relacionada con una temática del curso en ese preciso momento del año, el docente permitió que el estudiante que diligenciara el formulario tuviera una valoración complementaria en el resultado final evaluativo de la asignatura para el correspondiente periodo académico. Al término de la semana y en un nuevo encuentro con los grupos de estudiantes, se les permitió que reflexionaran sobre el desarrollo del trabajo y expusieran verbal y públicamente sus opiniones.

Los resultados obtenidos de esta actividad se analizaron mediante la selección de los cuestionarios que tenían información diligenciada y relacionada con el tema que se estaba indagando. La información fue procesada en una tabla de datos en Microsoft Excel y analizada con parámetros estadísticos descriptivos. Aspectos cualitativos fueron incorporados en el análisis de los datos empleando la técnica de análisis de contenido propuesta por Bardin, (2006) y la forma de escritura de los adolescentes fue respetada. Los animales citados se organizaron como etnoespecies, agrupándose seguidamente en orden taxonómico lineano (desde los invertebrados hasta los vertebrados).

\section{RESULTADOS Y DISCUSIÓN}

\section{Sobre el Uso de los Animales como Medicina}

De los 173 estudiantes a los que se les invitó a formar parte del estudio, sólo 68 respondieron satisfactoriamente el cuestionario. Quienes desarrollaron la tarea, citaron entre uno y cinco ejemplos de productos zooterapéuticos con diversos fines. En la matriz diseñada, se listaron 32 etnoespecies agrupadas en tres invertebrados y 29 vertebrados (según la escala de clasificación lineana). Las materias primas y tratamientos de enfermedades más referenciados son beber la cocción de un chulo para curar el "mojo" o "tocado de muerto" (13), emplear el veneno de las serpientes como antiofídico (12) y aplicar sobre el rostro de una persona la baba de caracol como limpiador facial para curar enfermedades 
cutáneas como manchas en la piel y acné (7). Dos estudiantes demostraron tener conocimientos médicos veterinarios (etnoveterinaria), aludiendo sobre algunos medicamentos que pueden suministrárseles a los caballos.

Los animales, sus partes o derivados de éstos son empleados para el tratamiento de 43 padecimientos o enfermedades, siendo las citaciones más frecuentes el "mojo" (18), antiofídico (16), asma (10), desnutrición o debilidad (10) y problemas cutáneos (7). El consumo de zooterapéuticos en algún momento de su vida fue confirmado por 23 estudiantes quienes respondieron afirmativamente (Cuadro 1; Anexo).

A pesar de que se esperaba que la acogida hubiese sido mucho más amplia de parte de los estudiantes para llevar a cabo la tarea, fue evidente que quienes decidieron resolver el cuestionario tienen algún tipo de conocimiento zooterapéutico y que ellos o sus familiares alguna vez han empleado animales y sus partes o derivados como medicina. Este hecho, denota que a pesar de que es más común encontrar que los conocimientos y prácticas médico tradicionales y populares estén referidos a las plantas, el uso de animales o sus productos en la medicina (zooterapeúticos) es cada vez más común y difundido en todas las comunidades del mundo (MARQUES, 1995; DA SILVA et al., 2004; COSTA NETO 2005; ALVES y ALVES, 2011; ALVES y ROSA, 2013).

Especialmente el "tocado de muerto", "mojo" o "enteco de difunto" es una de las enfermedades culturales más populares entre la población rural colombiana. Si bien en la resolución del cuestionario participaron familiares de los estudiantes que viven en el casco urbano de Yopal, muchos de ellos han resguardado y protegido este conocimiento que le han heredado sus ancestros rurales. Suárez y Pinzón (1988) realizaron un análisis de las posibles causas que le atribuían los indígenas a esta enfermedad en la que también los españoles aportaron creencias patológicas culturales que traían de Europa durante la conquista del nuevo mundo. Los autores mencionaban que el "tocado de muerto", que le da regularmente a la población infantil, podría estar fundamento en una falta de energía regida por la luna y no por el sol. Su explicación se basa en que el muerto toma la energía de los niños debilitándolos y provocándoles una desnutrición severa. En consecuencia, la gente aún cree que, dándole a beber caldo de chulo, palomo u otra bebida natural energizante, la vitalidad de la persona se regenera, evitándose que el infante se debilite o enferme a causa del robo de su energía vital.

Llama la atención que en una investigación sobre zooterapia en los mercados populares de Recife, Pernambuco, Brasil, realizada por Da Silva et al. (2004) a destacados comerciantes de productos medicinales, también hallaron que el urubú (el mismo chulo) era el animal más citado. Los vendedores mencionaron el empleo de plumas e hígado de este animal para el tratamiento del asma y el alcoholismo.

Se pudiera pensar que, al vencer el miedo al consumo de animales que usualmente son considerados sucios, feos o despreciables puede traer consigo mismo un vencimiento de los propios temores hacia factores aversivos, obteniéndose como beneficio o ganancia una "recarga" espiritual y de energía vital. Algo así como "si venzo a lo que le huyo también podría ganarle la partida a mis miedos y males internos".

El uso del veneno de la serpiente como antiofídico puede tener sus razones en las explicaciones que dan los profesores en la escuela durante las sesiones de clase. La preparación de sueros antiofídicos a partir de las mismas sustancias venenosas de las serpientes es una práctica tradicional que pasó a la industria farmacéutica y fue ampliamente difundida. Es un saber que provino de las esferas sociales más populares desde que se conocieron los efectos fisiológicos y la reacción del sistema inmunológico con el empleo de estas sustancias tóxicas en el cuerpo de los pacientes.

Entre tanto, el uso de "baba de caracol" como agente dermatológico también ha sido una práctica ampliamente difundida entre la sociedad. A través de diferentes medios publicitarios se presentan los grandes beneficios cosméticos alcanzados con este compuesto biológico. En tiendas naturistas, farmacias, mercados artesanales y populares y hasta en los mismos andenes de las calles comerciales de municipios y ciudades es fácil acceder a cremas cosméticas en la que se venden los componentes activos de la baba de caracol (ALCALDE y DEL POZO, 2008; ZARAGOZANO, 2017). De este modo se demuestra cómo el conocimiento popular acerca de los beneficios medicinales que puede aportar un animal, es reforzado por las campañas publicitarias comerciales que se realizan sobre dichos productos. El amplio uso de moluscos con fines terapéuticos en América Latina también fue registrado por Alves y Alves (2011).

\footnotetext{
${ }^{4}$ El enteco, mojo o tocado de muerto, se refiere a una carencia de energía provocada por el difunto y regida por la luna. El fallecido estaría tomando energía de
} los niños, siendo éstos los más susceptibles, obteniendo de éstos su energía, debilitándolos o provocándoles desnutrición (Suárez y Pinzón, 1988). 
Si bien la "baba de caracol" como exudado animal contiene entre otros compuestos químicos alantoína, ácido hialurónico, ácido glicólico, antiproteasas y antibióticos naturales que sirven en la actividad colagenasa (recambio de colágeno desnaturalizado), mejoramiento del citoesqueleto celular, disminución del espesor de la epidermis y los signos de elastosis cutánea y tiene una importante actividad antioxidante (ALCALDE y DEL POZO, 2008), es probable que muchas de las cremas que se ofrecen comercialmente y en sitios no autorizados, incumplan con las condiciones sanitarias y de fabricación que aseguren los efectos positivos que tiene verdaderamente el compuesto biológico en sus condiciones naturales.

Del estudio que se desarrolló con los estudiantes de Yopal, genera especial preocupación el consumo de carne de algunos vertebrados domésticos para "curar el hambre". Estas respuestas fueron interpretadas como el reflejo de un probable problema de seguridad alimentaria en las comunidades escolares investigadas. Y más aún si dichas comprensiones locales se relacionan con el "tocado de muerto" al ambas producir la desnutrición. Para la época en la que se desarrolló el estudio, el Estado colombiano no tenía un programa nacional de apoyo a la alimentación infantil.

\section{El diálogo intercultural en el aula de clase sobre el conocimiento escolar zooterapéutico}

Si bien en el presente estudio sólo se relacionan aspectos básicos de la zooterapia familiar, resulta importante reflexionar sobre el papel que tiene la escuela y el profesorado en generar espacios dentro y fuera del aula para que se conozcan, visibilicen y estudien las prácticas médicas tradicionales dentro de la comunidad escolar y la comunidad educativa en general. Ante la crisis del sistema de salud que afronta Colombia, los productos naturales vienen presentándose como alternativa para la sustitución de medicamentos que la industria farmacéutica coloca a precios relativamente altos en el mercado, y a los que la población más pobre no logra acceder (ZULUAGA, 2000; ALVES y DIAS, 2010). A partir de esta situación, el conocimiento de remedios y el funcionamiento de los sistemas médicos tradicionales están cobrando mayor vigencia, considerándose no sólo el importante y efectivo acervo médico biocultural con el que cuentan las poblaciones locales, sino también como punto de partida para continuar abriendo la discusión sobre su incorporación a la vida moderna y la trascendencia económica, política, antropológica, social, histórica y educativa que tiene este tipo de medicina en las sociedades contemporáneas (ALVES Y DIAS, 2010).

En este sentido, el diálogo intercultural en las aulas de clase y una reforma educativa a la enseñanza de las ciencias, juegan un papel preponderante al convertirse en estrategias efectivas para que se preste la importancia justa y necesaria a estos sistemas médicos desde la escuela, y no como simples conocimientos tradicionales y populares que frecuentemente son invalidados desde los mismos sistemas educativos y de salud nacionales. El diálogo intercultural es visto como una posibilidad didáctica en el aula de clase, convierte la opinión del otro en una oportunidad para democratizar el conocimiento, valorándolo, reconociéndolo y legitimándolo identitaria y culturalmente.

Para Haverkort et al. (2013), el diálogo intercultural, es comprendido como aquel cruce de saberes culturales que parten del principio de que todos los sistemas de conocimiento en el mundo poseen sus valores e importancia, que involucra un reconocimiento de la sabiduría de los pueblos nativos, con su propia epistemología, gnoseología y ontología como también de los sistemas de conocimiento moderno. Esta afirmación también se fundamenta en que se entiende que la ciencia se desarrolla a partir de sus propias dinámicas, interactúa y se retroalimentan de varios campos de conocimiento, donde se contempla el intercambio de métodos y resultados de investigación en esa búsqueda de respuestas para adaptar los propios paradigmas y sintetizar conjuntamente una pluralidad científica, donde la complementariedad puede coexistir con la inconmensurabilidad.

Según Ruiz y Villamar (2011), al existir un diálogo intercultural, prevalece el interés de los sujetos sociales en la interacción comunicativa que implica una disposición para escuchar y renovarse del otro. No se trata de imponer, vencer o inducir violentamente al otro a que acceda a la valoración o conocimiento ajeno, pues es, a partir de ese intercambio que fluyen las fuerzas racionales para la interacción comunicativa. De este modo, lo que se consigue es el resultado de una nueva síntesis, producto de cada sabiduría como efecto de un enriquecimiento mutuo. Se trata también de superar los fundamentalismos y universalizar su crítica, incluido el de occidente, como también su lógica económica y de conocimiento. Es considerar que la razón está influenciada por la cultura, que existen tantas verdades como culturas, estructuras sociales, intereses y creencias (CASTAÑO y MOLINA, 2012). 
Retomando las palabras de Zuluaga (2000) y contextualizándolas al ámbito escolar es importante entablar una relación de amistad caracterizada por el respeto a la diferencia, la eliminación de actitudes discriminatorias y el afán de compartir realidades que, aunque distintas son válidas. El sentido de la comunicación permanente en el aula de clase posibilita la reciprocidad y el fortalecimiento de las relaciones entre los mismos estudiantes o entre estudiantes y docentes (COLOMO-MAGAÑA 2011) y también consiste en la demarcación y no la anulación de saberes (COBERN y LOVING, 2001).

\section{Complementariedad de los Sistemas Médicos Tradicional y Moderno}

Una realidad existente en las escuelas de Latinoamérica y el Caribe es que se sigue replicando la anulación de los saberes que sean distintos a los contemplados en los lineamientos pedagógicos de los sistemas nacionales de educación. En el caso colombiano, en muchos casos el docente de aula no quiere correr el riesgo de incorporar otros contenidos diferentes a los planes de estudio para el área de conocimiento que imparte porque puede ser burlado, discriminado o sancionado socialmente a pesar de que, por otro lado, se le exige que innove en su práctica pedagógica. Especialmente en las escuelas urbanas, si un profesor de Ciencias Naturales y Educación Ambiental habla de chamanismo o de medicina indígena o campesina puede verse como si estuviera perdiendo el tiempo o sencillamente ser tildado de ser "loco", "brujo", o "desenfocado" pedagógicamente. Sigue prevaleciendo en el imaginario educativo urbano que hablar de culturas indígenas, afro o el campesinado, es referirse a algo interesante, extraño y remoto, pero no necesariamente incorporado a los planes de vida contemporáneos de la ciudad moderna. Esto, muy a pesar que entre las nuevas generaciones exista una preocupación por los aspectos ambientales, interculturales y la vida saludable.

Así como sucede con las prácticas agrícolas tradicionales en zonas rurales, los conocimientos ancestrales y modernos de la medicina se toman como complementarios, pues se necesitan de ambos para la síntesis de una perspectiva propia (MOLINA et al., 2011). No obstante, uno de los principales problemas que tenemos en los países latinoamericanos, es la falta de estudios que registren este conocimiento tradicional, por lo que una de las primeras tareas consistiría en recuperar esa memoria y tradición, labor que exige un ejercicio constante, disciplinado y respetuoso por el conocimiento local. Exige una tarea continua de incorporar en el currículo procesos y acciones concretas en las que se promuevan el fortalecimiento de la medicina tradicional, el diálogo permanente con los sabedores, curanderos, parteras, sobanderos, médicos tradicionales y una reconstrucción de la memoria biocultural con participación de los abuelos, todo con el fin en que redunde en la consecución de la transmisión de valores y técnicas a las nuevas generaciones (ZULUAGA, 2000).

El profesor Zuluaga (2000) agrega que, aunque no podemos negar los enormes avances científicos y tecnológicos en las diferentes áreas científicas, desde la medicina moderna se reconocen las limitaciones para dar solución a muchos de los problemas de salud que todavía aquejan a la humanidad. Al reconocer los saberes tradicionales asociados a la medicina, se enriquecería el sistema médico. Es innegable pensar que, por diversas circunstancias, amplios sectores de la población aún recurren a la medicina tradicional como primera opción.

\section{La Enseñanza de las Ciencias con una Visión Intercultural}

Una de las oportunidades que ofrece la educación en ciencias desde la interculturalidad es que el mismo diálogo promueve la unión y cohesión, la capacidad de enseñar y aprender a convivir con y desde la diferencia (COLOMO-MAGAÑA, 2011). Prevalece un reconocimiento de la existencia del "otro" como sujeto poseedor de una cultura diferente y el conocimiento de lo que esto significa en términos de semejanzas y diferencias con la cultura escolar, promoviéndose el intercambio de valores para la emergencia de situaciones más democráticas y solidarias (DIAZ-AGUADO, 2002 citado por LEIVA, 2008).

A partir de la enseñanza de las ciencias en el plano cultural se promueve la integración, la inclusión social y cultural. En las escuelas públicas y privadas, se hacen intentos por garantizar el derecho a una educación incluyente, equitativa y de calidad a través del reconocimiento de distintas cosmovisiones, lógicas, estéticas y formas de acceder al conocimiento con el fin de propiciar espacios para el desarrollo social (CASTAÑO, 2010; CASTAÑO y MOLINA, 2012), no obstante, si desde la estructura misma del sistema educativo esta necesidad no se transforma, lógicamente no se aplicaría a los diversos contextos locales y culturales, y mucho menos en las aulas de clase.

Una de las estrategias didácticas que generalmente se emplea cuando se enseña Ciencias Naturales en la escuela primaria es la de garantizar que no exista discriminación, exclusión y aislamiento de los 
estudiantes (MOLINA et al., 2011). Desde el campo de la interculturalidad no se puede promover la segregación, pues la escuela no puede ser reproductora de una realidad que, aunque existente, aparta lo diferente por el simple hecho de serlo o tener miedo a lo desconocido (COLOMO-MAGAÑA, 2011). La escuela y el aula de ciencias, por el contrario, deben convertirse en un lugar donde se deconstruyan lo estereotipado y las situaciones de desigualdad social y cultural, buscando promover la equidad (más que la igualdad) como principio de la educación intercultural (LEIVA, 2008).

Bajo esta perspectiva, la escuela y el aula de ciencias pasan de ser un espacio de discriminación y sanción, de transmisión y repetición, a constituirse en un lugar de encuentro y construcción intersubjetiva (CASTAÑO y MOLINA, 2012), pues uno de los retos que supone la incorporación de la interculturalidad en la educación en ciencias es precisamente el cambio de creencias, valores y actitudes entre todos los actores, desde los equipos administrativos locales de la educación, pasando por directivos y docentes hasta los estudiantes y sus familias.

Particularmente los docentes, en una aula multicultural se ven enfrentados a resolver conflictos personales que tienen que ver con sus principios, creencias, valores, comportamiento, y en general, en la forma como perciben y construyen el mundo (COLOMO-MAGAÑA, 2011), por lo que no es posible que profesores y estudiantes tengan manifestaciones racistas o xenófobas (LEIVA, 2008), y más cuando se trata de valorar la ciencia, la innovación o la tecnología como una construcción que nace desde la misma esencia de la cultura y que posibilita el desarrollo de una nación bajo principios epistemológicos propios.

Es necesario que el docente de ciencias genere conciencia hacia la importancia de la interculturalidad, demostrada en el cambio de sus acciones educativas y el respeto hacia la diversidad cultural (MOLINA et al., 2011). Ideas y opiniones de los estudiantes en relación con la forma de asumir la biología, ecología y el ambiente deben ser asumidas por el docente como una posibilidad para que todos reflexionen y repiensen su postura frente a los fenómenos que se estudian. La diversidad de imaginarios acerca de la vida, la naturaleza y el ambiente debe ser respetada, analizada, discutida, considerada y situada epistemológicamente en las aulas de clase, desde los primeros niveles de escolaridad de una persona.

Posiblemente los cambios que deban resolver los profesores no provengan únicamente del exterior, pues no necesariamente son los más efectivos, sino que deberán producirse al interior del aula a través de la reflexión e interacción con los educandos (COLOMO-MAGAÑA, 2011). Así pues, es necesario un esfuerzo para conocer el contexto cultural de los estudiantes, así como reflexionar sobre una nueva postura docente, que es el resultado de las interacciones que se producen entre los sujetos en las aulas. Para el educador estadunidense Donald Schön (2000), aprender a enseñar está relacionado con hacer, y para ello es necesario reflexionar sobre y en la acción pedagógica. El docente que reflexiona sobre sus acciones en el aula es capaz de promover mejoras en su formación y en la calidad de la enseñanza y el aprendizaje. Reflexiones sobre las interacciones que establece cómo los estudiantes pueden ir más allá del contexto de las aulas, creando y recreando estrategias que mejoren las relaciones con los estudiantes en las aulas y con sus familias.

Como docente, en un aula de ciencias con una posición de enseñanza y didáctica intercultural, no se puede seguir reproduciendo la postura vertical del "profesor, científico y sabio". Es claro que, cuando los profesores consideran la diversidad cultural en los procesos de enseñanza se enfrentan a múltiples polaridades, dualidades y contradicciones que envés de convertirse en un problema didáctico, lo que hacen es enriquecer las perspectivas y el panorama de la enseñanza e investigación en didáctica de las ciencias (MOLINA et al., 2011). Es asombrarse del conocimiento biológico local, para aprender con el otro.

El docente de ciencias debe estar expectante a las ideas y opiniones de sus estudiantes, pues ellos son portadores de saberes y experiencias culturales propias de su contexto. De igual forma, deben promoverse oportunidades para que los estudiantes comprendan los conocimientos científicos que son provenientes de la ciencia occidental, así como las influencias que estos pueden ejercer sobre los diversos medios sociales (BAPTISTA, 2014). El diálogo requiere de la escucha por parte del docente quien tiene la misión de establecer un puente entre los conocimientos tradicionales y los científicos para poder consolidar una ampliación del conocimiento sobre ese referente (MOLINA et al., 2011).

Si bien estos cambios dependen en gran medida de la voluntad docente, también existe la necesidad de formar maestros cuyo perfil profesional esté fundamentado en el desarrollo de competencias interculturales. En Colombia, las facultades de etnoeducación como de educación intercultural cubren 
en parte este déficit de las demás facultades de educación del país, sin embargo, los esfuerzos deberán aumentarse desde las universidades y escuelas normales superiores.

En esta dirección, Castaño (2010) se refiere a la tarea que ha venido ejerciendo la Universidad Pedagógica Nacional (Colombia) en formar docentes de Licenciatura en Biología con énfasis en ruralidad e interculturalidad. Desde 2008 en sus sedes Valle de Tenza (Boyacá), Puerto Asís (Putumayo) y La Chorrera (Amazonas), se han gestionado programas de pregrado de Licenciatura en Biología ${ }^{5}$ con enfoque intercultural en los que se ofrecen profundizaciones en líneas temáticas ajustadas a las necesidades locales (p.ej. educación rural, agroecología y silvicultura) y que atienden a la búsqueda de alternativas de solución a problemáticas regionales. Se abren posibilidades para que se ejecuten proyectos relacionados con los planes de vida de los estudiantes quienes son de origen indígena y campesino. Los currículos, que son construidos con los mismos estudiantes, propician el reconocimiento de identidades y la valoración y legitimación de sus saberes, costumbres y cultura. Desde luego, estas propuestas ayudan a construir profesionales más idóneos y capacitados para afrontar la multiculturalidad en la escuela.

\section{La Familia y la Comunidad: su protagonismo en la enseñanza de las ciencias naturales}

Otro de los factores clave en la educación intercultural es impulsar el valor que tiene la familia y la comunidad, pues esto indudablemente induce a que se piense en colectivo desde la identidad e individualidad de las personas, que se le halle sentido a pertenecer a un grupo que se caracteriza por ser diverso culturalmente y del cual se forma parte (COLOMO-MAGAÑA, 2011). Es una reivindicación, una protesta a los derechos culturales.

El trabajo de identificación y recuperación de las prácticas zooterapéuticas tradicionales en las aulas de clase, desde la escuela hacia la comunidad y viceversa, es un ejercicio de integración de las familias con la Institución Educativa. Estas actividades de formación cultural, exigen un relacionamiento positivo entre Escuela - Familia - Comunidad con los diferentes actores de la Comunidad Educativa; es un acercamiento y valoración de quienes forman parte del sistema médico local (parteras, sabios, curanderos, etc.) y los demás miembros de la comunidad que poseen un conocimiento empírico que les permite practicarlo y recrearlo permanentemente.

Desde la mirada educativa, el trabajo integral triangular Escuela-Familia-Comunidad favorece la creación de ambientes de aprendizaje diversos donde se recupera la tesis de que en la Institución Educativa no es el único lugar donde se aprende "lo importante". Y es aquí donde cobra mayor sentido hablar de la Escuela como una Comunidad de Aprendizaje.

\section{CONSIDERACIONES FINALES}

Los conocimientos zooterapéuticos entre los estudiantes y sus familias de las escuelas "El Paraíso" y "Carlos Lleras" del municipio de Yopal, Casanare, son una muestra de un acervo cultural médico poco explorado y que permanece vigente. Se registraron un total de 43 afecciones tratadas con prácticas zooterapéuticas, siendo las más citadas: el "mojo", uso de antiofídico para tratar el envenenamiento por serpientes, asma, desnutrición o debilidad y problemas cutáneos, para las que se emplean un total de 32 etnoespecies, agrupadas en tres invertebrados y 29 vertebrados (bajo el esquema de clasificación lineana).

El ejercicio realizado con los estudiantes de las escuelas públicas de Yopal, permitió reconocer la posibilidad de un diálogo intercultural e intergeneracional acerca del conocimiento zooterapéutico. No se buscó validar o anular conocimientos del otro sino compartirlos como un mecanismo de complementariedad, de manera que los estudiantes pudieran ampliar su universo de conocimientos y valorar los diferentes modos de solucionar padecimientos de salud, con la posibilidad de aplicar esos conocimientos en su contexto sociocultural.

La zooterapia es solo un pretexto investigativo para abordar otros temas que se pueden orientar desde el currículo de ciencias naturales y sociales con enfoque intercultural. Las percepciones y prácticas locales sobre el cambio climático, manejo del agua, uso del suelo, delimitaciones del territorio y aprovechamiento de la diversidad biológica y agrícola, son algunos de los temas que se pueden y deben abordar en las aulas de clase de ciencias.

La promoción de actividades como elaboración de narrativas, juegos, canciones, expresiones artísticas y culturales que favorezcan el diálogo intercultural en los salones de clases, pueden ser un

\footnotetext{
${ }^{5}$ La licenciatura en Colombia es entendida como la formación de profesorado para las escuelas Básica Primaria y Secundaria y Media.
} 
camino que permita promover la ampliación del universo de conocimientos que traen consigo los estudiantes, así como representar una forma de valorar sus propios saberes y los de sus compañeros.

El papel de la familia en la educación intercultural y aprendizaje de las ciencias es clave, pues es aquí donde los estudiantes adquieren los primeros conocimientos y tienen la oportunidad de formar parte de una colectividad con la que se identifican culturalmente. Además, los conocimientos sobre los fenómenos biológicos y naturales que poseen los estudiantes han sido adquiridos primero dentro del núcleo familiar o de la comunidad, por lo que la convivencia se vuelve fundamental no sólo para su aprendizaje sino también para su valoración y práctica.

La transversalidad curricular de los conocimientos y prácticas tradicionales asociadas a la naturaleza y el ambiente son un hecho ineludible que deberá seguir incursionando con mayor celeridad en los proyectos de educación propios e interculturales de las comunidades, así como en los currículos nacionales. Este hecho no sólo deberá ser para los pueblos donde la población mayoritaria tenga unas características culturales particulares, sino también para todos los establecimientos educativos del país, puesto que en Colombia se atiende educativamente desde un enfoque desde la inclusión social, cultural y diferencial, y eso no puede quedarse en discurso político, sino que tiene que materializarse de manera obligatoria en todos los niveles educativos.

\section{REFERENCIAS BIBLIOGRÁFICAS}

ALCALDE, M. T. y DEL POZO, A. Baba de Caracol. Offarm, v. 27, n. 9, p.118-120, 2008. Disponible en: https://www.elsevier.es/es-revista-offarm-4-pdf-13127394

ALCALDÍA DE YOPAL. 2007. Plan Básico de Ordenamiento Territorial de Yopal (2000-2011). Acuerdo Municipal No. 12. Municipio de Yopal, Casanare, 2007, 187p.

ALVES, R. R. y ALVES, H. N. The faunal drugstore: animal-based remedies used in traditional medicines in Latin America, Journal of Ethnobiology and Ethnomedicine, v. 7, n. 9, p. 1-43, 2011.

ALVES, R. R. y DIAS, T. L. Usos de invertebrados na medicina popular no Brasil e suas implicações para conservação. Tropical Conservation Science, n. 3, v. 2, p. 159-174, 2010.

ALVES R. R. y ROSA, I. E. Animals in Traditional Folk Medicine. Springer-Verlag Berlin Heidelber, 2013, p. 492

ARGUETA, A. El diálogo de saberes, una utopía realista. En: Argueta, A., Corona-M., E. y Hersch, P. (Eds.) Saberes colectivos y diálogo de saberes en México. México, D.F: UNAM, CRIM, Cuernavaca y Universidad Iberoamericana, Puebla, 2011, p. 495-510.

BALL, S. J. y BOWE, R. Subject departments and the "implementation" of National Curriculum policy: an overview of the issues. Journal of Curriculum Studies, v. 24, n 2, p. 97-115, 1992.

BAPTISTA, G. C. S. Do cientificismo ao diálogo intercultural na formação do professor e ensino de ciências. Interacções, n. 31, p. 28-53, 2014.

BARDIN, L. Análise de conteúdo. Lisboa: Edições 70, 2006. (Obra original publicada em 1977).

BELTRÁN-CUARTAS, A.M., SILVA, N.M., LINARES, E.L. y CARDONA, F.A. 2010. La etnobotánica y la educación geográfica en la comunidad rural Guacamayas, Boyacá, Colombia. UniPluriVersidad, v. $10, \quad$ n. $3, \quad$ p. $1-11, \quad 2010.4$ Disponible en: https://revistas.udea.edu.co/index.php/unip/article/view/9586. Acceso en: 13 nov. 2013.

CANEN, A. Educação multicultural, identidade nacional e pluralidade cultural: tensões e implicações curriculares. Cadernos de Pesquisa, n. 111, p. 135-150, 2000.

CASTAÑO, N. 2010. Formación de docentes en biología con pertinencia y en contexto, desde una perspectiva intercultural. Una invitación a reconocer- nos... lo propio en los otros y lo de los otros en lo propio. En: Congreso Nacional de Investigación en Educación en Ciencias y Tecnología, Cali, Valle del Cauca, Colombia. II Memorias del Congreso Nacional de Investigación en Educación en Ciencias y Tecnología. Cali: Asociación Colombiana para la Investigación en Ciencia y Tecnología, Universidad Distrital Francisco José de Caldas, 2010. Disponible en: http://portales.puj.edu.co/dhermith/Ponencias\%20Finales_congreso_Educyt/

Formacion $\% 20$ de $\% 20$ docentes $\% 20 \mathrm{en} \% 20$ biologia $\% 20$ con $\% 20$ pertinencia $\% 20 \mathrm{y} \% 20$ en $\% 20$ conte.pdf. Acceso. Acceso: 10 nov 2010.

CASTAÑO, N. C. y MOLINA, A. Concepciones acerca de la vida, enseñanza de la biología y diversidad cultural. La construcción del conocimiento educativo para un futuro humano. En: III Congreso Internacional y VIII Nacional de Investigación en Educación y Formación Docente, Bogotá, Colombia. Libro de Memorias del Congreso Internacional y VIII Nacional de Investigación en Educación y Formación Docente, Bogotá: ASCOFADE/ Universidad de Antioquia, Universidad 
Distrital Francisco José de Caldas/ Universidad de Valle/ Universidad Pedagógica Nacional/ Universidad Pedagógica Nacional de México, 2012, p. 641-656.

COBERN, W. Constructivism and non-western science education research. International Journal of Science Education, Routledge, v. 4, n. 3, p. 287-302, 1996.

COBERN W. y LOVING, C. Defining science in a multicultural world: implications for science education. Science Education, v. 85, n. 1, p. 50-67, 2001.

COBERN, W. Apples and oranges: a rejoinder to Smith and Siegel. Science Education, v. 13, n. 6 , p. 583-589, 2004.

COLOMO-MAGAÑA, E. El profesor ante la diversidad educativa. Revista Digital Transversalidad Educativa, n.51, p. 15-35, 2011.

CONTCEPI (Comisión Nacional para el Trabajo y Concertación de la Educación para los Pueblos Indígenas). Perfil del sistema educativo indígena propio SEIP. Documento preliminar. Bogotá: Organización Nacional Indígena de Colombia/ Autoridades Indígenas de Colombia/ Organización de los Pueblos Indígenas de la Amazonia Colombiana/ Confederación Indígena Tayona 2012, 141p. Disponible en: http://cms.onic.org.co/wp-content/ uploads/2012/06/CONTCEPI-perfil-SEIP-versionII2012.pdf. Acceso en: 12 nov 2013.

COSTA NETO, E. Animal-based medicines: biological prospection and the sustainable use of zootherapeutic resources. Anais da Academia Brasileira de Ciências, v. 77, n. 1, p 33-43, 2005.

DA SILVA, M.L., ALVES, A.G. y ALMEIDA, A. Zooterapia no Recife (Pernambuco): uma articulação entre as práticas e a história. Biotemas, v. 17, n. 1, p. 95 - 116, 2004.

DUREAU, F. y GOUESET, V. El proceso migratorio y sus consecuencias sobre el poblamiento de las ciudades petroleras: realidades y representaciones colectivas en el caso de las ciudades de Casanare, Colombia. Scripta Nova, Revista Electrónica de Geografía y Ciencias Sociales, v. 94, n. 76, 2001. Disponible en: http://www.ub.edu/geocrit/sn-94-76.htm. Accesado en: 12 nov 2010.

HAVERKORT, B., MILLAR, D., SHANKAR, D. y DELGADO, F. Relación entre diferentes comunidades de conocimiento. El rechazo, la sustitución, la complementariedad y el diálogo intercientífico. En: Haverkort, B., Delgado, F., Shankar, D. y Millar, D. (Eds.) Hacia el diálogo intercientífico, construyendo desde la pluralidad de visiones del mundo, valores y métodos en diferentes comunidades de conocimiento. La Paz: Agruco, 2013, p. 21-42.

INSTITUCIÓN EDUCATIVA CARLOS LLERAS RESTREPO. Proyecto Educativo Institucional: ciencia, virtud y futuro. Documento técnico, Yopal, 2011, 60p.

INSTITUCIÓN EDUCATIVA EL PARAÍSO. Proyecto Educativo Institucional: ciencia, paz y trabajo. Documento técnico. Yopal, 2010, 71p.

LEIVA, J.J. Interculturalidad, gestión de la convivencia y diversidad cultural en la escuela: un estudio de las actitudes del profesorado. Revista Iberoamericana de Educación, v. 46, n. 2, p. 1-14, 2008.

MARQUES, J. G. W. Pescando Pescadores: uma etnoecologia abrangente no baixo São Francisco alagoano. São Paulo: NUPAUB-USP, 1995, 304p.

MARTÍNEZ-RODRÍGUEZ, M. Ethnobotanical knowledge acquisition among tsimane' children in the Bolivian Amazon. 2009, 141p. Tesis (Doctor of philosophy)- University of Georgia, Georgia, U.S.A.

MINISTERIO DE EDUCACIÓN NACIONAL. Estándares Básicos de Competencias en Ciencias Naturales y Ciencias Sociales - Formar en Ciencias: jel desafío! Lo que necesitamos saber y saber hacer. Serie de Guías No. 7. Ministerio de Educación Nacional. Bogotá D.C. 2001, 48p. Disponible: https://www.mineducacion.gov.co/1759/articles81033_archivo_pdf.pdf. Acceso: 13 sep 2014.

MINISTERIO DE EDUCACIÓN NACIONAL. Plan Sectorial 2010-2014. Documento No. 9. Bogotá D.C: Ministerio de Educación Nacional, 2010, 110p.

MINISTERIO DE EDUCACIÓN NACIONAL. Derechos Básicos de Aprendizaje en Ciencias Naturales. Subdirección de Referentes y Evaluación de la Calidad Educativa. Bogotá D.C: Ministerio de Educación Nacional, 2016a.

MINISTERIO DE EDUCACIÓN NACIONAL. Matrices de Referencia en Ciencias Naturales. Subdirección de Referentes y Evaluación de la Calidad Educativa. Bogotá D.C: Ministerio de Educación Nacional, 2016b.

Ministerio de Educación Nacional. Derechos Básicos de Aprendizaje en Ciencias Sociales. Subdirección de Referentes y Evaluación de la Calidad Educativa. Bogotá D.C: Ministerio de Educación Nacional, 2016c. 
MINISTERIO DEL INTERIOR. 2014. Decreto 1953 mediante el cual "se crea un régimen especial con el fin de poner en funcionamiento los Territorios Indígenas respecto de la administración de los sistemas propios de los pueblos indígenas hasta que el Congreso expida la ley de que trata el artículo 329 de la Constitución Política”. República de Colombia. Octubre 7 de 2014.

MOLINA, A., MOJICA, L., MOSQUERA, C.J., MARTÍNEZ, C.A., REYES, J.D., CIFUENTES, M.C. y PEDREROS, R.I. Pontes no ensino da ciência e diversidade cultural, Perspectivas dos profesores. En: VIII Encontro de Pesquisa em Educação em Ciências - ENPEC, I Congreso Iberoamericano de Investigación en Enseñanza de las Ciencias CIIEC. 2011, Campinas. Atas do VIII Encontro de Pesquisa em Educação em Ciências - ENPEC, I Congreso Iberoamericano de Investigación en Enseñanza de las Ciencias CIIEC, Campinas: ABRAPEC, 2011.

MORENO, R.E. Las plantas medicinales de la Escuela La Laguna. Eduteka, 2012. Disponible en: http://www.eduteka.org/proyectos.php/2/13100. Acceso en: 20 ago 2014.

ROJAS, H., MORENO, M. y CASTILLO, N. Casanare, un departamento que debe conocerse. Gobernación de Casanare. Comité Pedagógico. Núcleo Educativo 2. Colombia: Editorial Talleres Gráficos Ltda. Tunja, 1997.

RUIZ, M. L. P.; VILlamaR, A. A. Saberes indígenas y diálogo intercultural. Cultura y Representaciones Sociales, v. 5, n. 10, p.31-56 2011. Disponible en: http://www.scielo.org.mx/scielo.php?script=sci_arttext\&pid=S2007-81102011000100002. Acceso en: 16 sep 2020.

SCHÖN, D. A. Educando o Profissional Reflexivo: um novo design para o ensino e a aprendizagem. Trad.Roberto Cataldo Costa. Porto Alegre: Artmed, 2000, 256p.

SILVA, T. T. Documentos de Identidade: uma introdução às teorias do currículo. 2. ed. Belo Horizonte: Autêntica, 2003.

SUÁREZ, R. y PINZÓN, C. Salud y estrategias comunitarias. Maguaré, v. 6, n. 6-7, p. 69-100, 1988.

VALLEJO, O. Yopal, ciudad siglo XXI. Segunda Edición. El Yopal, 2011.

VERDE, A., VALDÉS, A., RIVERA, D., FAJARDO, J., OBÓN, C., RUÍZ-GALlARDO, J.R., BENLLOCH, V., CIUDAD, R., NÚÑEZ, P. y PIERA, A.. La Etnobiología como materia transversal en el currículo de educación secundaria. Una experiencia en Castilla La-Mancha, España: Ensayos, Revista de la Facultad de Educación de Albacete, n. 24, p. 149-162, 2009.

ZARAGOZANO, J.F. El caracol como alimento y como terapia. Boletín de la Sociedad de Pediatría de Aragón, La Rioja y Soria, v. 47, n. 3, p. 67-72, 2017.

ZULUAGA, G. Plantas medicinales y etnobiología: un enfoque ético para la etnobiología. Boletín de Antropología, v. 6, n. 6, p. 21-32, 2000. 
Anexo

Cuadro 1. Usos zooterapéuticos registrados en las escuelas del Yopal, Casenare, Colombia. (En el apartado se respetaron las transcripciones de los estudiantes).

\begin{tabular}{|c|c|c|c|c|}
\hline $\begin{array}{l}\text { Familia } \\
\text { taxonómica }\end{array}$ & $\begin{array}{l}\text { Nombre } \\
\text { tradicional }\end{array}$ & Producto utilizado & Preparación & Indicador émico \\
\hline Hirudinidae & $\begin{array}{l}\text { Sanguijuela/ } \\
\text { Sandijuela? }\end{array}$ & Saliva (baba) & ¿? & $\begin{array}{l}\text { "Ayuda para la circulación de la sangre y } \\
\text { aliviar los dolores" (ATPA, } 12 \text { años) }\end{array}$ \\
\hline \multirow{4}{*}{ Helicidae } & \multirow{4}{*}{ Caracol } & \multirow[t]{2}{*}{ Baba } & $\begin{array}{l}\text { Aplicar sobre la } \\
\text { piel }\end{array}$ & $\begin{array}{l}\text { "La baba de caracol sirve para limpiar la } \\
\text { cara" (NDGG, } 12 \text { años); "Para la cara, Para } \\
\text { quitar los barros" (FJM, } 13 \text { años); "Para los } \\
\text { barros" (EJA, } 13 \text { años) }\end{array}$ \\
\hline & & & $\begin{array}{l}\text { Aplicar sobre la } \\
\text { herida }\end{array}$ & $\begin{array}{l}\text { "Para la cicatris" (PAJ, } 10 \text { años); "Se le } \\
\text { echa la vaba a la persona para la erida en la } \\
\text { erida se le echa" (JSP, } 12 \text { años). }\end{array}$ \\
\hline & & Piel & $\begin{array}{l}\text { Frotar sobre la } \\
\text { parte afectada }\end{array}$ & $\begin{array}{l}\text { "Se restriega en la piel del niño para curarle } \\
\text { las heridas" (FJJF, } 12 \text { años) }\end{array}$ \\
\hline & & Caparazón & ¿? & "Sasco para las manchas" (MFA, 13 años) \\
\hline \multirow{3}{*}{ Apidae } & \multirow{3}{*}{ Abeja } & \multirow{2}{*}{ Miel } & Se bebe la miel & " Se toma para la gripa" (NAL, 9 años) \\
\hline & & & $\begin{array}{l}\text { Frotar sobre la } \\
\text { parte afectada }\end{array}$ & $\begin{array}{l}\text { "Se saca la miel y se restrega para la } \\
\text { adtritis" (BR, } 12 \text { años); }\end{array}$ \\
\hline & & Panal & ¿? & $\begin{array}{l}\text { "Con una cucharada de miel para la tos" } \\
\text { (JMV, } 12 \text { años) }\end{array}$ \\
\hline \multirow{7}{*}{ Gadidae } & Bacalao & Animal completo & ¿? & "Para la gripa" (MDS, 10 años) \\
\hline & \multirow{5}{*}{ Raya } & \multirow[t]{2}{*}{ Grasa } & $\begin{array}{l}\text { Se derrite la grasa } \\
\text { se deja reposar y } \\
\text { luego se da a beber } \\
\text { al enfermo }\end{array}$ & $\begin{array}{l}\text { "Para la tos" (XTG, } 11 \text { años); "tomado para } \\
\text { los frios de pulmón" (AIN, } 9 \text { años) }\end{array}$ \\
\hline & & & Bebida & $\begin{array}{l}\text { "Se toma y sirve para la tos y curar } \\
\text { heridas" (VIG, } 13 \text { años) }\end{array}$ \\
\hline & & Manteca & ¿? & $\begin{array}{l}\text { "La mateca de raya para el pujo" (AKB, } 14 \\
\text { años) }\end{array}$ \\
\hline & & Grasa del hígado & $\begin{array}{l}\text { Se ingiere una vez } \\
\text { extraída y } \\
\text { conservada la grasa } \\
\text { en forma de } \\
\text { manteca }\end{array}$ & $\begin{array}{l}\text { "Se extrae el hígado luego se derrite se } \\
\text { conserva la manteca en un frasco se toma } \\
\text { para la toz tibia" }\end{array}$ \\
\hline & & Hígado & ¿? & $\begin{array}{l}\text { "El dolor de la picadura de ella misma" } \\
\text { (JYV, } 11 \text { años) }\end{array}$ \\
\hline & Tiburón & Grasa & $\begin{array}{l}\text { Se derrite la } \\
\text { manteca }\end{array}$ & $\begin{array}{l}\text { "Se derrite la manteca del tiburon para la } \\
\text { tos ferina" (JAS, } 12 \text { años) }\end{array}$ \\
\hline \multirow[b]{2}{*}{ Bufonidae } & \multirow[b]{2}{*}{ Sapo } & Animal completo & $\begin{array}{l}\text { Frotar el animal } \\
\text { completo sobre la } \\
\text { parte afectada }\end{array}$ & $\begin{array}{l}\text { "Para curar algunas infecciones se pasa el } \\
\text { sapo" (ILM, } 11 \text { años) }\end{array}$ \\
\hline & & Buche & ¿? & $\begin{array}{l}\text { "Cuando una persona se le pone roja la } \\
\text { pierna se pone el sapo en esa parte pa que } \\
\text { se le pase. El sapo se muere" (JED, } 12 \\
\text { años) }\end{array}$ \\
\hline \multirow{3}{*}{ Colubridae } & \multirow{3}{*}{$\begin{array}{l}\text { Culebra/ } \\
\text { serpiente }\end{array}$} & Veneno & ¿? & $\begin{array}{l}\text { "Sirve para sacar otro veneno" (ATPA, } 13 \\
\text { años); "Para quitar el veneno de otras } \\
\text { serpientes" (AJM, } 12 \text { años); "Inyectándolo } \\
\text { para sanar las mordidas de serpiente" (SCR, } \\
11 \text { años }\end{array}$ \\
\hline & & Sangre & ¿? & ¿? \\
\hline & & Hiel & Consumo directo & $\begin{array}{l}\text { "El remedio se utiliza para la mordedura de } \\
\text { culebra, se utiliza matando el animal se } \\
\text { mide } 3 \mathrm{~cm} \text { depues del ano se corta se extrae } \\
\text { la hiel y ce toma" (PAGS, } 12 \text { años) }\end{array}$ \\
\hline Crotalidae & $\begin{array}{l}\text { Culebra } \\
\text { cascabel/ } \\
\text { serpiente } \\
\text { cascabel }\end{array}$ & Colmillos & ¿? & $\begin{array}{l}\text { "Se le apreta los colmillos saca el veneno } \\
\text { para sacarle la picadura de culebra" (JSP, } \\
12 \text { años) }\end{array}$ \\
\hline
\end{tabular}




\begin{tabular}{|c|c|c|c|c|}
\hline & & Animal completo & $\begin{array}{l}\text { Secar el animal, } \\
\text { tostarlo, triturarlo y } \\
\text { luego dar en una } \\
\text { bebida }\end{array}$ & $\begin{array}{l}\text { "La vicecaron y la tostaron para luego } \\
\text { molerla y darle de tomar" }\end{array}$ \\
\hline & & Veneno & ¿? & $\begin{array}{l}\text { "Para combatir la mordedura y el veneno } \\
\text { de las serpientes" (JYV, } 11 \text { años) }\end{array}$ \\
\hline & Cobra & Animal completo & ¿? & "Pasta para el cáncer" (MDS; 10 años) \\
\hline \multirow{8}{*}{ Iguanidae } & Iguana & Uñas & ¿? & $\begin{array}{l}\text { "Sirve para que cresca el pela" (ATPA, } 12 \\
\text { años) }\end{array}$ \\
\hline & \multirow{3}{*}{$\begin{array}{l}\text { Tortuga/ } \\
\text { morroco }\end{array}$} & Carne & $\begin{array}{l}\text { Cocción de todo la } \\
\text { carne }\end{array}$ & $\begin{array}{l}\text { "Cocinada solo carne se utiliza tambien } \\
\text { para el mojo" }\end{array}$ \\
\hline & & Caparazón & Lamer la caparazón & $\begin{array}{l}\text { "Lamiendolo para los gases" (SCR, } 11 \\
\text { años) }\end{array}$ \\
\hline & & Sangre & $\begin{array}{l}\text { Bañar la persona } \\
\text { con la sangre }\end{array}$ & $\begin{array}{l}\text { " Se baña la persona con la sangre y se } \\
\text { utiliza para los niños que tienen hielo" } \\
\text { (HDLC, } 11 \text { años) }\end{array}$ \\
\hline & \multirow{3}{*}{$\begin{array}{l}\text { Guio, } \\
\text { "huivo"? }\end{array}$} & \multirow{3}{*}{ Manteca } & $\begin{array}{l}\text { Aplicar la manteca } \\
\text { del animal sobre las } \\
\text { heridas }\end{array}$ & $\begin{array}{l}\text { "Echandose en la herida para las peladuras" } \\
\text { (AYGR, } 11 \text { años) }\end{array}$ \\
\hline & & & ¿? & $\begin{array}{l}\text { "Para los síntomas de la tos" (JYV, } 11 \\
\text { años) }\end{array}$ \\
\hline & & & En bebida & "Se toma para la gripa" (VIG, 13 años) \\
\hline & Mato & Cuero & $\begin{array}{l}\text { ?/Se seca y luego } \\
\text { se muele }\end{array}$ & $\begin{array}{l}\text { "para el romatismo" (AYGR, } 11 \text { años); " } \\
\text { Se seca y luego se muele para el } \\
\text { romatismo" (JAS, } 12 \text { años) }\end{array}$ \\
\hline \multirow{5}{*}{ Phasianidae } & \multirow{5}{*}{ Gallina } & Animal completo & Caldo & $\begin{array}{l}\text { " Caldo para la mujer cuando recién tiene } \\
\text { bebe" (bs, } 10 \text { años) }\end{array}$ \\
\hline & & Manteca & $\begin{array}{l}\text { Aplicar sobre la } \\
\text { herida }\end{array}$ & $\begin{array}{l}\text { "Hechandosela en la erida para zanar" } \\
\text { (MBM, } 11 \text { años) }\end{array}$ \\
\hline & & Huevo & ¿? & "Para los orzuelos" (CA, 9 años) \\
\hline & & Cáscara de huevo & $\begin{array}{l}\text { Se da una bebida a } \\
\text { base de la harina de } \\
\text { la cáscara de huevo } \\
\text { mezclada con leche }\end{array}$ & $\begin{array}{l}\text { "Cascara se muele que quede como harina } \\
\text { se da en la leche como calcio" (ADR, } 9 \\
\text { años) }\end{array}$ \\
\hline & & Clara de huevo & $\begin{array}{l}\text { Aplicación directa } \\
\text { sobre el cuero } \\
\text { cabelludo }\end{array}$ & $\begin{array}{l}\text { "Para fortalecer el cabello se aplica en el } \\
\text { cabello" (ADR, } 9 \text { años) }\end{array}$ \\
\hline Anatidae & Pato & Embrión & ¿? & $\begin{array}{l}\text { "Para vitaminas y cuidar el cabello" (YE, } \\
13 \text { años) }\end{array}$ \\
\hline \multirow{4}{*}{ Cathartidae } & \multirow{4}{*}{ Chulo } & $\begin{array}{l}\text { Sangre, animal } \\
\text { entero }\end{array}$ & $\begin{array}{l}\text { A) se bebe la } \\
\text { sangre; b) se } \\
\text { prepara un caldo } \\
\text { con el resto del } \\
\text { animal; c) se coloca } \\
\text { a hervir y se licúa. }\end{array}$ & $\begin{array}{l}\text { "Se mata el animal se toma la sangre y con } \\
\text { el resto se ase un caldo y se toma" (ANND, } \\
11 \text { años); " lo ponen ha hervir y lo licuan y } \\
\text { lo toman asi" }\end{array}$ \\
\hline & & Animal completo & $\begin{array}{l}\text { Cocción de todo el } \\
\text { animal }\end{array}$ & $\begin{array}{l}\text { "Se cosina se deja enfriar el agua se baña el } \\
\text { niño y se enbuelbe en una sabana blanca } \\
\text { esto sirve para el mojo o tocado de muerto" } \\
\text { (PAGS, } 12 \text { años); para un niño que tenia } \\
\text { mojo y se hirvio el chulo luego se toma el } \\
\text { agua"se baña la persona con el agua y se } \\
\text { utiliza para el hielo y el mojo" (HDLC, } 11 \\
\text { años); "cuando los bebes van al sementerio } \\
\text { les da una enfermedad que se llama hielo se } \\
\text { calienta y se baña el niño" (JSP, } 12 \text { años); } \\
\text { se hierbe el agua se mete el chulo se baña el } \\
\text { niño para quitar vavo muerto" (AIM, } 9 \\
\text { años) }\end{array}$ \\
\hline & & Sangre & $\begin{array}{l}\text { Baño con el agua } \\
\text { del animal, luego se } \\
\text { envuelve la persona } \\
\text { con una sábana } \\
\text { blanca }\end{array}$ & "Para la tensión del cuerpo" \\
\hline & & Carne & $\begin{array}{l}\text { Cocción de todo el } \\
\text { animal }\end{array}$ & $\begin{array}{l}\text { "Se cocina y se come para el cancer" } \\
\text { (CCC }, 11 \text { años) }\end{array}$ \\
\hline
\end{tabular}




\begin{tabular}{|c|c|c|c|c|}
\hline & & Animal completo & $\begin{array}{l}\text { Cocción de todo el } \\
\text { animal }\end{array}$ & $\begin{array}{l}\text { "Lo cogieron lo pelaron lo calentaron como } \\
\text { caldo y se lo dieron a las personas que no } \\
\text { tenian animo y nutrisión" (JSP, } 12 \text { años) }\end{array}$ \\
\hline Corvidae & Cuervo & Animal completo & ¿? & "Para la artritis" (BSN, 12 años) \\
\hline \multirow{5}{*}{ Columbidae } & \multirow{5}{*}{ Paloma } & Sangre & $\begin{array}{l}\text { A) beber la sangre; } \\
\text { b) beber la sangre } \\
\text { mezclada con vino } \\
\text { "sonson" }\end{array}$ & $\begin{array}{l}\text { "Se lo toma y se utiliza para la anemia" } \\
\text { (XTG, } 11 \text { años); "se degolla el animal se } \\
\text { recibe la sangre en una copa se rebuelve } \\
\text { con vino sonson y se toma para la anemia" } \\
\text { (PAGS, } 12 \text { años) }\end{array}$ \\
\hline & & ¿? & $\begin{array}{l}\text { Preparación de } \\
\text { caldo }\end{array}$ & $\begin{array}{l}\text { "Se mata se abre y se pone en la cabeza y } \\
\text { luego se hace un caldo para la devilidad" } \\
\text { (CCC, } 11 \text { años); "se toma caliente la sangre } \\
\text { para la desnutrición" (LDGS, } 12 \text { años); } \\
\text { "para abrir el apetito" (TMN, } 12 \text { años) }\end{array}$ \\
\hline & & ¿? & ¿? & "Para el asma" (AO; 11 años) \\
\hline & & Animal completo & ¿? & $\begin{array}{l}\text { "Se raja por la mitad para darle fuerza de } \\
\text { fertilidad a la mujer en la matris" (JSP, } 12 \\
\text { años) }\end{array}$ \\
\hline & & ¿? & ¿? & $\begin{array}{l}\text { "Para la fuerza para que los niños caminen" } \\
\text { (CAF, } 9 \text { años) }\end{array}$ \\
\hline Dasypodidae & $\begin{array}{l}\text { Cachicamo, } \\
\text { armadillo }\end{array}$ & Sangre & $\begin{array}{l}\text { Se toma la sangre } \\
\text { directamente }\end{array}$ & $\begin{array}{l}\text { "Se le sacó la sangre y se toma" (ANND, } \\
10 \text { años) }\end{array}$ \\
\hline \multirow{2}{*}{ Muridae } & \multirow{2}{*}{ Rata } & Sangre & i? & $\begin{array}{l}\text { "Para sanar mordidas o desinfectante" } \\
\text { (LTZ, } 13 \text { años) }\end{array}$ \\
\hline & & Grasa & Masajear el pecho & $\begin{array}{l}\text { "Se utilizó en forma de masaje. Sirve para } \\
\text { la tos" (NYS, } 12 \text { años) }\end{array}$ \\
\hline \multirow{4}{*}{ Agoutidae } & Lapa & Hiel & & $\begin{array}{l}\text { "Para la mordedura de serpiente" (LNH, } 13 \\
\text { años) }\end{array}$ \\
\hline & \multirow{3}{*}{$\begin{array}{l}\text { Chigüiro, } \\
\text { chigüire }\end{array}$} & \multirow{2}{*}{ Hiel } & $\begin{array}{l}\text { Secar la hiel y } \\
\text { tomarla con agua }\end{array}$ & $\begin{array}{l}\text { "Para la diabetes se seca la hiel y se toma } \\
\text { con agua" (LDGS, } 12 \text { años) }\end{array}$ \\
\hline & & & Cocción & $\begin{array}{l}\text { "Se cocina para la migraña o dolores } \\
\text { articulares" (AJM, } 12 \text { años) }\end{array}$ \\
\hline & & Carne & Ingestión directa & "para curar el hambre" (BSN, 12 años) \\
\hline \multirow{5}{*}{ Equidae } & \multirow{4}{*}{ Caballo } & Crin & $\begin{array}{l}\text { Se amarra un pelo } \\
\text { de la crin del } \\
\text { caballo alrededor } \\
\text { del mezquino hasta } \\
\text { que se caiga } \\
\end{array}$ & ¿? \\
\hline & & Testículos & Ingestión directa & $\begin{array}{l}\text { "Se lo comen se utiliza para las estrías" } \\
\text { (XTG, } 11 \text { años); "se capa el caballo se le } \\
\text { echan los testigulos en una botella con } \\
\text { aguardiente se soban las pierna de para } \\
\text { arriba sirve para la vena várice" (PAGS, } 12 \\
\text { años) }\end{array}$ \\
\hline & & & $\begin{array}{l}\text { Se aplica sobre la } \\
\text { parte afectada }\end{array}$ & $\begin{array}{l}\text { "Le hechamos neugon en las orejas para } \\
\text { que no le sigan los moscos infectando la } \\
\text { parte enferma" (JAE, } 11 \text { años) }\end{array}$ \\
\hline & & & Anemia equina & $\begin{array}{l}\text { "Sirve para la vacuna de la anemia equina" } \\
\text { (JYV, } 11 \text { años) }\end{array}$ \\
\hline & Burro & Orejas & ¿? & $\begin{array}{l}\text { "Se toma en jugo para libiar los parásitos" } \\
\text { (SCR, } 11 \text { años) }\end{array}$ \\
\hline Canidae & Perro & Baba & $\begin{array}{l}\text { Frotar sobre la } \\
\text { parte afectada }\end{array}$ & $\begin{array}{l}\text { "El perro le lanbe en la hernia para que le } \\
\text { sane rapido! (FJJG, } 12 \text { años) }\end{array}$ \\
\hline \multirow[b]{2}{*}{ Felidae } & \multirow{2}{*}{ Gato } & Cola & $?$ & "Para un remedio en los ojos" \\
\hline & & Grasa & Beber la grasa & $\begin{array}{l}\text { "Para el asma un vaso de manteca de } \\
\text { cachicamo" (AYGR, } 11 \text { años) }\end{array}$ \\
\hline \multirow[b]{2}{*}{ Bovidae } & \multirow[b]{2}{*}{ Vaca } & \multirow[b]{2}{*}{ Leche } & ¿? & "para la diarrea" (LTZ, 13 años) \\
\hline & & & Beber & $\begin{array}{l}\text { "se calienta una herradura que quede roja } \\
\text { se introduce en la leche se da a beber para } \\
\text { levantar a la persona devil" (KMN, } 12 \\
\text { años) }\end{array}$ \\
\hline
\end{tabular}




\begin{tabular}{|c|c|c|c|c|}
\hline & & Tuétano & $\begin{array}{l}\text { Frotar sobre la } \\
\text { parte afectada con el } \\
\text { preparado de los } \\
\text { tuétanos en alcohol }\end{array}$ & $\begin{array}{l}\text { "para sobarse los huesos y para el dolor de } \\
\text { cabeza le echavan alcohol y se sobaban" } \\
\text { (AYGR, } 11 \text { años); "se utiliza para sobarse" } \\
\text { (VIG, } 13 \text { años) }\end{array}$ \\
\hline & & ¿? & ¿? & $\begin{array}{l}\text { "para el mojo cuando un niño es tocado de } \\
\text { muerto" (MDS, } 10 \text { años) }\end{array}$ \\
\hline & & Cascos & $\begin{array}{l}\text { Aspirar los cascos } \\
\text { calientes }\end{array}$ & $\begin{array}{l}\text { "lo calentaron y que lo oliera" (YAH, } 11 \\
\text { años) }\end{array}$ \\
\hline & & Pata & & $\begin{array}{l}\text { "cuando los niños están débiles" (SY, } 9 \\
\text { años) }\end{array}$ \\
\hline & & Pajarilla & $\begin{array}{l}\text { Licuar con mora y } \\
\text { se da en bebida }\end{array}$ & $\begin{array}{l}\text { "se licua con mora se les da a los bebes } \\
\text { cuando están deviles" (JED, } 12 \text { años) }\end{array}$ \\
\hline & & Manteca & $\begin{array}{l}\text { Aplicar la manteca } \\
\text { del animal sobre las } \\
\text { heridas }\end{array}$ & "se aplica en los nacidos" (KMN, 12 años) \\
\hline & & Menudo & $?$ & "para los niños con el mojo" (NGC, 9 años) \\
\hline & & Carne & Ingestión directa & $\begin{array}{l}\text { "se utiliza para curar el hambre" (ILM, } 11 \\
\text { años) }\end{array}$ \\
\hline & Toro & Cascos & ¿? & $\begin{array}{l}\text { "para la hemorragia" (MFA, } 13 \text { años); } \\
\text { "para quitar la hemorragia" (MGT, } 11 \text { años) }\end{array}$ \\
\hline & Chivo & ¿? & Barbas & $\begin{array}{l}\text { Se colocan sobre una pañoleta las barbas } \\
\text { del chivo con brandy y panela y se colocan } \\
\text { debajo del coto para que desinflamen las } \\
\text { glándulas }\end{array}$ \\
\hline & & Grasa (manteca) & $\begin{array}{l}\text { Frotar el vientre } \\
\text { con la manteca }\end{array}$ & $\begin{array}{l}\text { "Para sobarse la barriga para el dolor de } \\
\text { barriga se fritaba y se ponia en un } \\
\text { recipiente" (AYGR, } 11 \text { años) }\end{array}$ \\
\hline Suidae & $\begin{array}{l}\text { Marrano/ } \\
\text { cerdo }\end{array}$ & & $\begin{array}{l}\text { Frotar sobre las } \\
\text { articulaciones }\end{array}$ & $\begin{array}{l}\text { "Se utilizaba para sobarse del dolor de } \\
\text { huesos" (VIG, } 11 \text { años) }\end{array}$ \\
\hline & & Carne & Ingestión directa & "Para curar el hambre" (LCN, 11 años) \\
\hline
\end{tabular}

